\title{
Possible Anandamide and Palmitoylethanolamide involvement in human stroke
}

\author{
Marcello Naccarato*1, Daniela Pizzuti2 ${ }^{2}$ Stefania Petrosino ${ }^{3}$, Marco Simonetto ${ }^{1}$, Laura Ferigo ${ }^{1}$, Fabio Chiodo Grandi ${ }^{1}$, \\ Gilberto Pizzolato ${ }^{1}$ and Vincenzo Di Marzo 3
}

\begin{abstract}
Background: Endocannabinoids (eCBs) are ubiquitous lipid mediators that act on specific (CB1, CB2) and non-specific (TRPV1, PPAR) receptors. Despite many experimental animal studies proved eCB involvement in the pathogenesis of stroke, such evidence is still lacking in human patients. Our aim was to determine eCB peripheral levels in acute stroke patients and evaluate their relationship with clinical disability and stroke volume.

Methods: A cohort of ten patients with a first acute (within six hours since symptoms onset) ischemic stroke and a group of eight age- and sex-matched normal subjects were included. Groups were also matched for metabolic profile. All subjects underwent a blood sample collection for anandamide (AEA), 2-arachidonoylglycerol (2-AG) and palmitoylethanolamide (PEA) measurement; blood sampling was repeated in patients on admission (T0), at 6 (T1) and 18 hours (T2) thereafter. Patients neurological impairment was assessed using NIHSS and Fugl-Meyer Scale arm subitem (FMSa); stroke volume was determined on $48 \mathrm{~h}$ follow-up brain CT scans. Blood samples were analyzed by liquid chromatography-atmospheric pressure chemical ionization-mass spectrometry.

Results: 1)TO AEA levels were significantly higher in stroke patients compared to controls. 2)A significant inverse correlation between TO AEA levels and FMSa score was found. Moreover a positive correlation between T0 AEA levels and stroke volume were found in stroke patients. TO PEA levels in stroke patients were not significantly different from the control group, but showed a significant correlation with the NIHSS scores. T0 2-AG levels were lower in stroke patients compared to controls, but such difference did not reach the significance threshold.

Conclusions: This is the first demonstration of elevated peripheral AEA levels in acute stroke patients. In agreement with previous murine studies, we found a significant relationship between AEA or PEA levels and neurological involvement, such that the greater the neurological impairment, the higher were these levels.
\end{abstract}

\section{Background}

During the last decade numerous studies have addressed the role of the endocannabinoid (eCB) system in different pathological conditions. Endocannabinoids (eCBs), e.g. anandamide (AEA) and 2-arachidonoylglycerol (2-AG), are lipid mediators synthesized "on demand" that inhibit neurotransmitter (glutamate and GABA) release and modulate neuroinflammation by activating specific $\mathrm{CB}_{1}$ (highly expressed in the CNS, where they mediate the psychotropic effects of $\Delta^{9}$-tetrahydrocannabinol) and $\mathrm{CB}_{2}$ (expressed by immune cells, including brain resident

\footnotetext{
* Correspondence: marcello.naccarato@katamail.com

1 Department of Medical, Technological and Translational Sciences, Neurology Unit, University of Trieste, Strada di Fiume 447, 34100 Trieste (TS), Italy Full list of author information is available at the end of the article
}

microglial cells) receptors, respectively. Cannabinoid receptor-inactive eCB-related molecules, e.g. palmitoylethanolamide (PEA), also exert neuroprotective effects[13], presumably by preventing mast cell degranulation [4], and directly activating peroxisome proliferator-activated receptor (PPAR)- $\alpha$ [5], or by enhancing the effects of AEA on cannabinoid receptors, transient receptor potential vanilloid type-1 (TRPV1) channels and PPAR- $\gamma$ receptors [6].

Previous murine and cell culture studies on stroke and hypoxia postulated a neuroprotective role of eCBs, given their ability to decrease NMDA-mediated toxicity in vascular penumbra through a $\mathrm{CB}_{1}$-mediated mechanism [7]. Increases of AEA content, of the AEA biosynthetic precursors (e.g. $\mathrm{N}$-acyl phosphatidylethanolamines), and of 
$\mathrm{CB}_{1}$ receptors expression in ischemic brain regions of murine stroke models have been described [8-11]. $\mathrm{CB}_{1}$ knockout mice develop larger stroke volumes than wildtype animals, with consequent increased post-stroke disability and mortality [12]. In addition, $\mathrm{CB}_{1}$ agonist administration was associated with a decrease of infarct volume and with an improvement of clinical symptoms in stroketreated mice [13]. Interestingly, there is evidence that also low-doses of $\mathrm{CB}_{1}$ receptor antagonists, such as rimonabant, reduce infarct volume in stroke models $[9,10,14,15]$, possibly by enhancing TRPV1-mediated actions [14]. It has been also suggested that part of the neuroprotective effects of $\mathrm{CB}_{1}$ receptor agonists in stroke is due to their capability of lowering body temperature, and that $\mathrm{CB}_{1}$, as opposed to $\mathrm{CB}_{2}$, receptors might otherwise play a counterprotective role in cerebral ischemia [15].

Indeed, also $\mathrm{CB}_{2}$ receptors have been implicated in the pathogenesis of stroke. Since such receptors are particularly expressed on activated microglia and peripheral immune cells (mastcells, macrophages and lymphocytes), they may act by modulating the inflammatory response to stroke [16], which is triggered 24-48 hours after symptoms onset and is mainly responsible for the delayed neuronal death [17]. Indeed $\mathrm{CB}_{2}$ agonists administration was associated with a reduction of infarct volume and neurological impairment in murine models of stroke and cerebral ischemia $[15,18]$. However, such evidence is still lacking in humans, despite the fact that a single case report described an increase of AEA and PEA content in the ischemic hemisphere of a stroke patient [19].

Aim of this study was to evaluate the possible involvement of the eCB system in stroke patients, by measuring plasma AEA, 2-AG, and PEA levels in the acute phase of the disease and by correlating $\mathrm{eCB}$ and PEA plasmatic levels with measures of neurological impairment and volume of the ischemic brain tissue.

\section{Methods Subjects}

10 patients (Group A; M/F = 5/5; mean age $70 \pm 13$ years) affected by a first ischemic stroke involving the Middle Cerebral Artery (MCA) territory with at least arm impairment and symptoms onset $\leq 6$ hours before admission, and 8 age-matched healthy volunteers (Group B; M/ $F=4 / 4$; mean age $70 \pm 12$ years) were enrolled. Patients with other neuropsychiatric diseases, substance and/or alcohol abuse, or systemic inflammatory diseases were excluded. The study was approved by the local institutional ethical committee and each participant (or relative, in case of aphasia/impaired consciousness) gave written informed consent in accordance with the Declaration of Helsinki. On admission (T0), patients underwent CT brain scanning, evaluation of neurological impairment with the NIHSS [20] and the Fugl-Meyer arm subitem scale (FMSa) [21], calculation of the Body Mass Index (BMI), blood sampling for routine laboratory analysis (Cholesterol, Triglycerides, Fasting Blood Glucose) and for eCB measurements. Blood sampling for eCB determination was repeated at 6 hours (T1) and 18 hours (T2) after admission. A follow-up brain CT scan was performed 48 hours after admission.

Control subjects (Group B), after written informed consent, underwent blood sampling for eCB and laboratory analysis; their BMI was also calculated.

\section{Endocannabinoid determination}

Blood was collected in EDTA and immediately centrifuged at $400 \mathrm{~g}$ for $30 \mathrm{~min}$ at room temperature. Extraction, purification of AEA, 2-AG and PEA, and their quantification by isotope-dilution liquid chromatography-atmospheric pressure chemical ionization-mass spectrometric analysis were performed as previously described [22].

\section{Brain CT analysis}

Twenty-eight contiguous $5 \mathrm{~mm}$-thick axial slices, covering the whole brain, were obtained for each patient with a multislice TOSHIBA AQUILION scanner, with $120 \mathrm{kV}$, $250 \mathrm{~mA}$ and $2 \mathrm{~s}$ acquisition time (Toshiba Medical Systems). Thereafter, using Analyze 4.5 (Biomedical Imaging Resource, Mayo University) and a semi-automated routine, two blinded examiners (FCG and GP) calculated the total lesion volume in $\mathrm{mL}$, as the sum of the stroke area in each slice multiplied by slice thickness (Fig. 1).

\section{Statistical analysis}

Using the SPSS 13.0 statistical software package (SPSS Inc.), inter-group differences were evaluated with the Mann-Whitney U test, while the Spearman rho test was used to evaluate correlations between eCBs plasma levels and the clinical and radiological data.

\section{Results}

Groups were comparable with regard to age, gender, BMI, and laboratory findings (Tab. 1). Mean AEA, PEA, and 2AG blood levels in the control group (Group B) were similar to previously reported values [22-25] and correlated with total cholesterolemia and BMI.

At T0, AEA plasma levels in the stroke patient group were significantly higher than in the control group (Tab. 1 and Fig. 2; Mann-Whitney U test: $\mathrm{p}<0.05$ ). In addition, AEA levels at T0 showed a significant inverse relationship with the FMSa scores (Fig 3; Spearman rho $=-0.819, \mathrm{p}=$ 0.004 ), so that patients with greater neurological impairment (lower FMSa score) had higher AEA levels. There was also a positive correlation between T0 plasma AEA levels and the volume of the ischemic brain region on $\mathrm{CT}$ scans (Spearman rho $=0.667, \mathrm{p}<0.05$ ). 


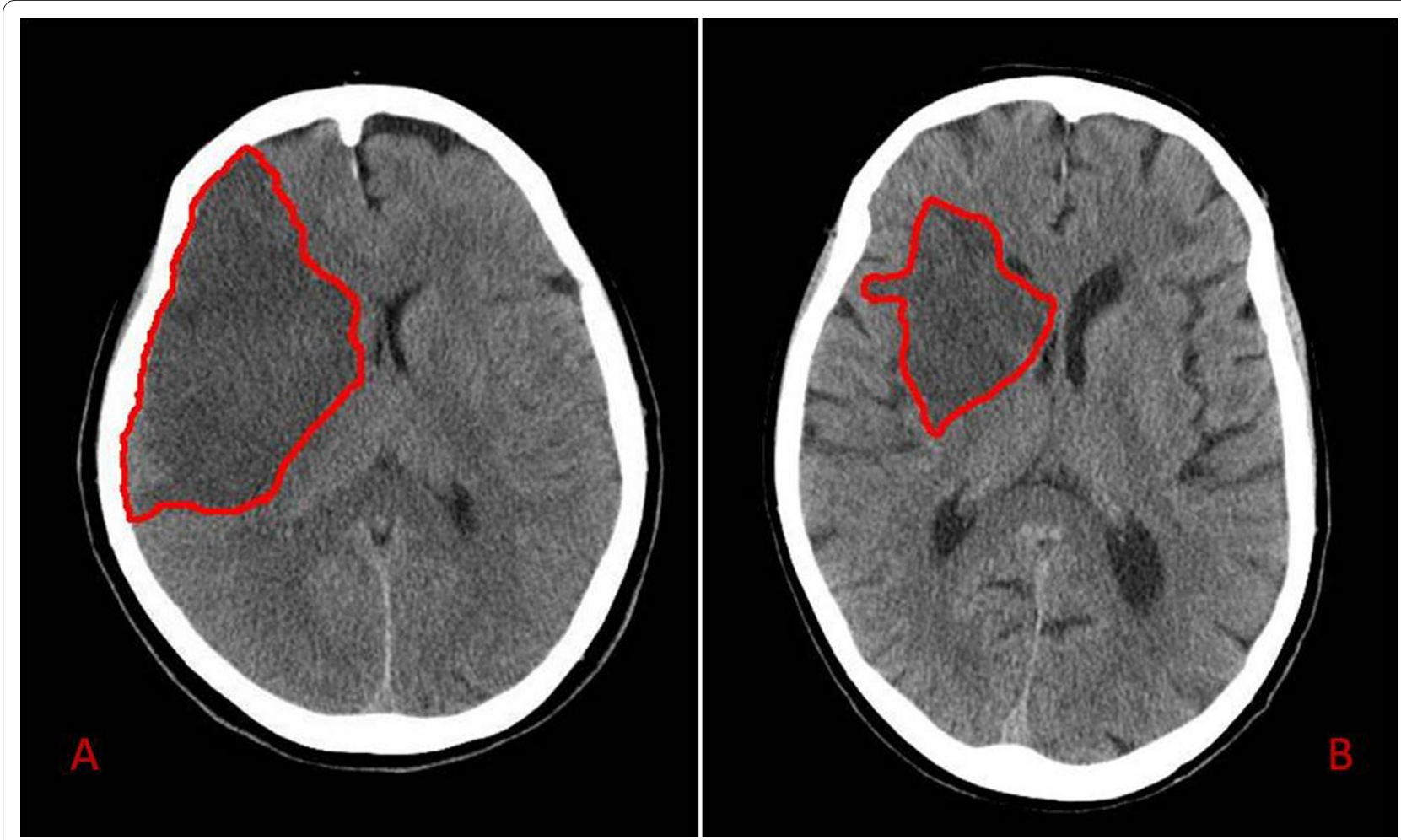

Figure 1 Follow-up brain CT scans of two stroke patients. Two examples of CT scans of two stroke patients at 48 hours since admission. The red outline circumscribes the ischemic area. The patient in panel A had a total occlusion of right middle cerebral artery, presenting with left hemiparesis and hemianopia (Female; Age = 80 yrs.; T0 AEA = 7.31 pmol/lipid mg; TO NIHSS = 17; T0 aFMS score = 12). The patient in panel B was admitted for a left hemiparesis due to a partial occlusion in right middle cerebral artery territory (Female, Age $=82$ yrs.; TO AEA $=3.93 \mathrm{pmol} / \mathrm{lipid} \mathrm{mg} ; \mathrm{TO} \mathrm{NIHSS}=10$; T0 aFMS score $=18$ ).

No significant differences between groups in PEA plasmatic levels were observed. Nevertheless, there was a significant correlation between PEA levels at T0 and the NIHSS scores (Fig. 4; Spearman rho $=0.823, \mathrm{p}=0.003$ ), such that the greater the neurological impairment, the higher the PEA levels.

Plasma 2-AG levels were lower in stroke patients, but group differences did not reach statistical significance.

No significant differences in AEA or PEA content were observed at later time-points ( $\mathrm{T} 1$ and $\mathrm{T} 2$ ) between groups (Tab. 1).

\section{Discussion}

Previous studies showed an involvement of the eCB system in various neurological conditions [26]. To our knowledge, this is the first study addressing the potential involvement of eCBs and a related mediator in acute stroke in a cohort of human patients.

Since eCBs have been proposed to act as local mediators and not as circulating hormones, the physiopathological consequences in stroke of the observed alterations in eCB plasma levels cannot be easily inferred. Nevertheless, there are several reports of changes in plasma endocannabinoid levels in patients affected by various neurological and neuropsychiatric conditions, including multiple sclerosis [27], Huntington's chorea [28], migraine [29,30], schizophrenia [22], depression [31] and anorexia nervosa [23]. Therefore, although one would expect that such conditions alter endocannabinoid signalling mostly in the brain, there is clearly a "spill-over" effect to the peripheral circulation, the extent of which often reflects, as in the present case, the severity of CNS pathology. Accordingly, endocannabinoid blood levels usually are 1-2 orders of magnitude lower than brain levels. Interestingly, a recent review by Maccarrone's group emphasized the relationship between brain diseases and endocannabinoid peripheral dysregulation, showing that peripheral eCBs could be a marker of CNS pathologies [32].

Peripheral AEA levels at T0 were significantly increased in stroke patients and showed a positive correlation with neurological disability and stroke volume. Likewise, plasma PEA levels showed a significant correlation with neurological disability. There was also a trend to reduced 2-AG levels in stroke patients. On the other hand, AEA and PEA levels at later time-points were not different between groups. These observations parallel the findings of a single case study in a patient with hemi- 
Table 1: Demographic and laboratory data of stroke patients and control subjects.

\begin{tabular}{|c|c|c|c|}
\hline & Group A & Group B & $\mathbf{p}$ \\
\hline $\operatorname{Sex}(M / F)$ & $5 / 5$ & $4 / 4$ & n.s. \\
\hline Age yrs. (mean $\pm S D)$ & $70 \pm 13$ & $70 \pm 12$ & n.s. \\
\hline $\mathrm{NIHSS}$ score (median $\pm S D$; range) & $22 \pm 10 ; 3-29$ & - & - \\
\hline Fugl-Meyer Scale-arm score (median $\pm S D$; range) & $11.50 \pm 9 ; 8-44$ & - & - \\
\hline $\mathrm{CT}$ ischemic area Volume $(\mathrm{mL}$; mean $\pm S D)$ & $76.10 \pm 43.46$ & - & - \\
\hline Body Mass Index (mean $\pm S D$ ) & $25.20 \pm 2.74$ & $24.63 \pm 2.39$ & n.s. \\
\hline Cholesterol (mmol/L - mean $\pm S D)$ & $11.69 \pm 2.31$ & $11.64 \pm 2.04$ & n.s. \\
\hline Triglycerides (mmol/L - mean $\pm S D$ ) & $6.97 \pm 2.33$ & $6.82 \pm 1.00$ & n.s. \\
\hline Blood Glucose (mmol/L - mean $\pm S D)$ & $5.61 \pm 0.71$ & $5.77 \pm 0.74$ & n.s. \\
\hline AEA TO (pmol/lipid mg - mean $\pm S D)$ & $3.42 \pm 2.71$ & $1.81 \pm 1.53$ & 0.026 \\
\hline AEA T1 (pmol/lipid mg - mean $\pm S D)$ & $2.87 \pm 2.34$ & - & n.s. \\
\hline AEA T2 (pmol/lipid mg - mean \pm SD) & $3.11 \pm 2.72$ & - & n.s. \\
\hline PEA TO (pmol/lipid mg - mean $\pm S D)$ & $2.47 \pm 0.96$ & $2.05 \pm 0.31$ & n.s. \\
\hline PEA T1 (pmol/lipid mg - mean $\pm S D)$ & $2.28 \pm 1.01$ & - & n.s. \\
\hline PEA T2 (pmol/lipid mg - mean $\pm S D)$ & $2.17 \pm 0.67$ & - & n.s. \\
\hline 2-AG T0 (pmol/lipid mg - mean $\pm S D)$ & $3.42 \pm 7.22$ & $6.80 \pm 9.50$ & n.s. \\
\hline 2-AG T1 (pmol/lipid mg- mean $\pm S D)$ & $4.65 \pm 4.98$ & - & n.s. \\
\hline 2-AG T2 (pmol/lipid mg - mean $\pm S D)$ & $3.29 \pm 3.10$ & - & n.s. \\
\hline
\end{tabular}

Demographic, clinical, radiological data and endocannabinoid or PEA plasma levels in stroke patients (Group A, at T0, T1, and T2 time-points) and control subjects (Group B). $p=p$ values (Mann-Whitney $U$ test); $n . s .=$ not significant.

spheric stroke in which AEA and PEA contents were precociously increased in microdyalisates of tissue surrounding the ischemic lesion [19]. They also parallel several studies showing early increases of AEA and PEA, but reduced 2-AG, in ischemic brain regions in animal models of global and focal cerebral ischemia [33]. Interestingly, Maccarrone's group found an increase of CSF

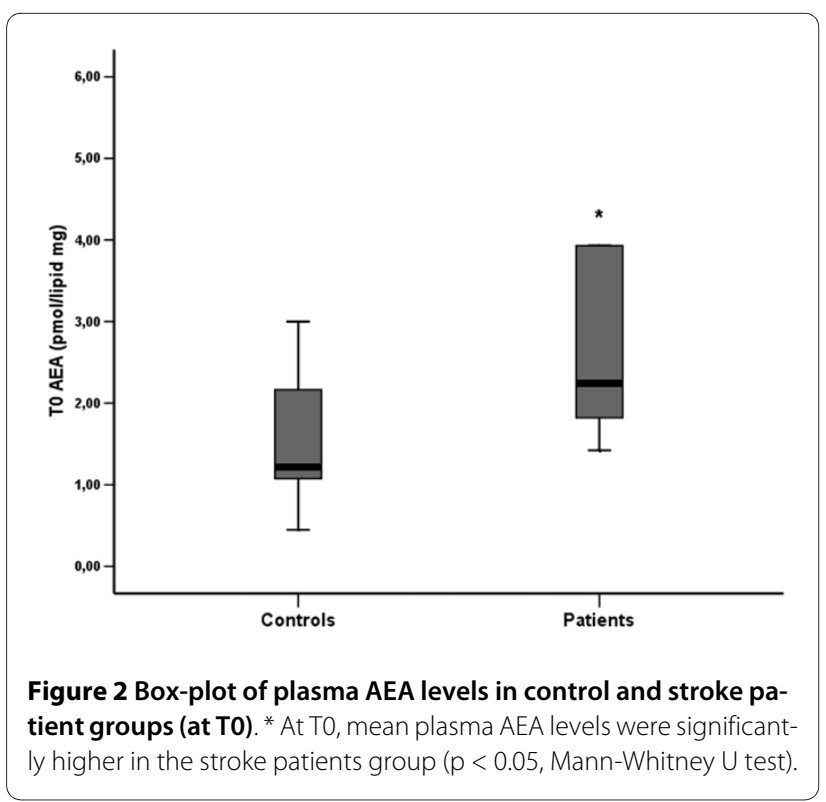

and plasmatic AEA, but not 2-AG, content in multiple sclerosis patients during relapses [27]. In addition, the same group recently demonstrated that an increase of striatal AEA levels may result in a decrease of 2-AG biosynthesis, thus suggesting an inverse relationship between AEA and 2-AG levels, under certain conditions [34].

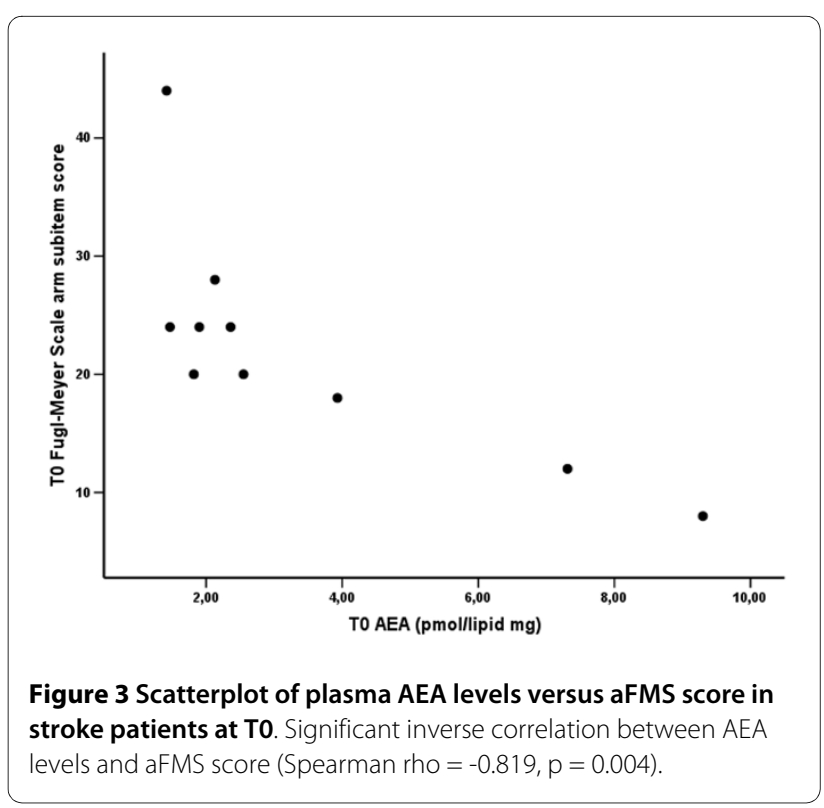




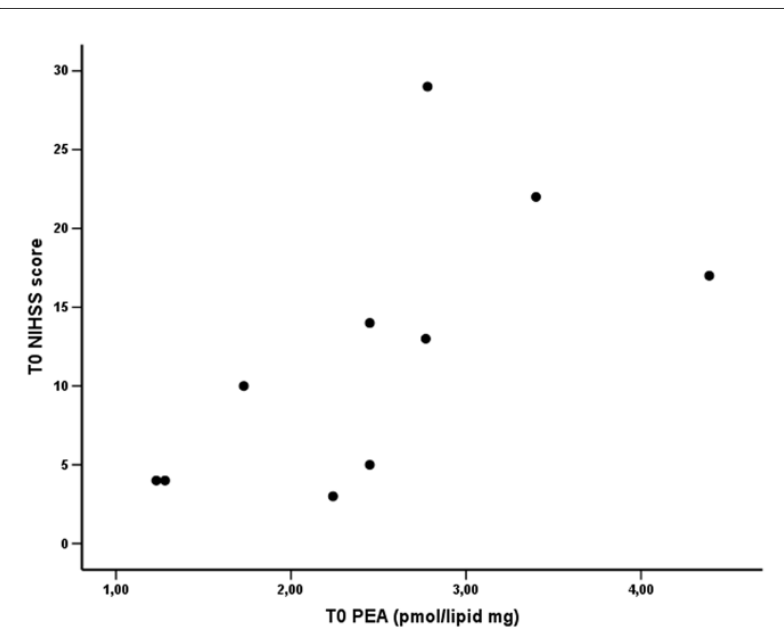

Figure 4 Scatterplot of plasma PEA levels versus NIHSS score in stroke patients at T0. Significant direct correlation between PEA levels and NIHSS score (Spearman rho $=0.823, p=0.003$ ).

The overall effect of activation of the eCB system in response to ischemia is not yet fully elucidated. In our study, plasma AEA and PEA contents in stroke patients correlated with neurological disability, but this relationship does not per se imply a potential neuroprotective effect. Recently, Schomacher reported that i.p. administration of AEA or PEA in rats 30 min after transient MCA occlusion significantly reduced the size of the infarcted tissue [3]. Since AEA acts as an endogenous agonist for $\mathrm{CB}_{1}$ and $\mathrm{CB}_{2}$ receptors, its neuroprotective effect may be mediated by inhibition of citotoxic glutamate release and by reducing the probability of the opening of voltageoperated calcium channels through $\mathrm{CB}_{1}$-mediated mechanisms. Indeed, $\mathrm{CB}_{1}$-knockout mice develop larger stroke volumes than wild-type animals [12]. $\mathrm{CB}_{2}$ mediated effects have also been implicated in stroke and in this case the eCBs may act by modulating the inflammatory response that contributes to the delayed neuronal death [33]. Such response might also be reduced by elevation of PEA levels, since PEA plays anti-inflammatory and neuroprotective effects via several potential mechanisms $[3,35]$.

\section{Conclusions}

Our findings in stroke patients show an early increase in plasma AEA content which correlates with neurological disability and infarct volume. Our study extends previous observations from the case report by Schäbitz and colleagues [19], showing a relationship between plasma AEA content and neurological disability. However, our present findings do not allow to determine, for instance, whether the increase in AEA levels is a consequence of stroke and exerts a possible protective effect, or whether it is part of the mechanisms leading to neurological damage during stroke. Therefore, further studies are warranted to address the therapeutic potential of eCB system modulation in stroke patients, as already explored in several (pre)clinical studies on other neurological diseases, such as multiple sclerosis, Alzheimer's disease, and brain trauma $[26,36]$. Finally, the role and mechanism of action of PEA in neuroprotection against cerebral ischemia also needs to be more deeply investigated.

\section{Competing interests \\ The authors declare that they have no competing interests.}

\section{Authors' contributions}

MN was involved in experimental planning, clinical data collection, data analysis and manuscript writing. DP was involved in experimental planning and endocannabinoid determination. SP performed endocannabinoid determination and contributed to data analysis. MS and LF were involved in clinical evaluation, clinical data collection and manuscript editing. FCG performed neuroimaging data analysis, contributed to data collection and manuscript editing. GP contributed to experimental planning, neuroimaging data analysis and manuscript editing. VDM contributed to experimental planning, data analysis and manuscript writing.

All authors read and approved the final manuscript.

\section{Acknowledgements and Funding}

This study has been funded by Giuliani Farmaceutici s.p.a. and Epitech Italia. The authors would thank the Radiology (Prof. M. Cova) and Immunotransfusional Departments (Dott. L. Uxa) of the Azienda Ospedaliero-Universitaria of Trieste for their kind help in providing the radiological data and contributing to the blood samples analysis.

\section{Author Details}

1Department of Medical, Technological and Translational Sciences, Neurology Unit, University of Trieste, Strada di Fiume 447, 34100 Trieste (TS), Italy, 2Department of Surgical and Gastroenterological Sciences, University of Padova, Via Giustiniani 5, Padova (PD), Italy and 'Endocannabinoid Research Group, Institute of Biomolecular Chemistry, National Research Council, Via Campi Flegrei 34, Pozzuoli (NA), Italy

Received: 6 April 2010 Accepted: 14 May 2010

Published: 14 May 2010

\section{References}

1. Skaper SD, Buriani A, Dal Toso R, Petrelli L, Romanello S, Facci L, Leon A: The ALlamide palmitoylethanolamide and cannabinoids, but not anandamide, are protective in a delayed postglutamate paradigm of excitotoxic death in cerebellar granule neurons. Proc Natl Acad Sci USA 1996, 93:3984-3989.

2. Lombardi G, Miglio G, Varsaldi F, Minassi A, Appendino G: Oxyhomologation of the amide bond potentiates neuroprotective effects of the endolipid N-palmitoylethanolamine. J Pharmacol Exp Ther 2007, 320:599-606.

3. Schomacher M, Müller HD, Sommer C, Schwab S, Schäbitz WR: Endocannabinoids mediate neuroprotection after transient focal cerebral ischemia. Brain Res 2008, 1240:213-220.

4. Facci L, Dal Toso R, Romanello S, Buriani A, Skaper SD, Leon A: Mast cells express a peripheral cannabinoid receptor with differential sensitivity to anandamide and palmitoylethanolamide. Proc Natl Acad Sci USA 1995, 92:3376-3380.

5. Lo Verme J, Fu J, Astarita G, La Rana G, Russo R, Calignano A, Piomelli D: The nuclear receptor peroxisome proliferator-activated receptor-alpha mediates the anti-inflammatory actions of palmitoylethanolamide. Mol Pharmacol 2005, 67:15-19.

6. Costa B, Comelli F, Bettoni I, Colleoni M, Giagnoni G: The endogenous fatty acid amide, palmitoylethanolamide, has anti-allodynic and antihyperalgesic effects in a murine model of neuropathic pain: 
involvement of $\mathrm{CB}(1)$, TRPV1 and PPARgamma receptors and neurotrophic factors. Pain 2008, 139:541-550.

7. Kim SH, Won SJ, Mao XO, Jin K, Greenberg DA: Molecular mechanisms of cannabinoid protection from neuronal excitotoxicity. Mol Pharmacol 2006, 69:691-696

8. Muthian S, Rademacher DJ, Roelke CT, Gross GJ, Hillard CJ: Anandamide content is increased and CB1 cannabinoid receptor blockade is protective during transient, focal cerebral ischemia. Neuroscience 2004, 129:743-750.

9. Berger C, Schmid PC, Schabitz WR, Wolf M, Schwab S, Schmid HHO: Massive accumulation of $\mathrm{N}$-acylethanolamines after stroke. Cell signalling in acute cerebral ischemia? J Neurochem 2004, 88:1159-1167.

10. Jin $K$, Mao XO, Goldsmith PC, Greenberg DA: CB1 cannabinoid receptor induction in experimental stroke. Ann Neurol 2000, 48:257-261.

11. Amantea D, Spagnuolo P, Bari M, Fezza F, Mazzei C, Tassorelli C, Morrone LA, Corasaniti MT, Maccarrone M, Bagetta G: Modulation of the endocannabinoid system by focal brain ischemia in the rat is involved in neuroprotection afforded by 17 beta-estradiol. FEBS J 2007 , 274:4464-4475.

12. Parmentier-Batteur S, Jin K, Mao XO, Xie L, Greenberg DA: Increased severity of stroke in CB1 cannabinoid receptor knock-out mice. $J$ Neurosci 2002, 22:9771-9775.

13. Nagayama T, Sinor AD, Simon RP, Chen J, Graham SH, Jin K, Greenberg DA: Cannabinoids and neuroprotection in global and focal cerebral ischemia and in neuronal cultures. J Neurosci 1999, 19:2987-2995.

14. Pegorini S, Braida D, Verzoni C, Guerini-Rocco C, Consalez GG, Croci L, Sala $\mathrm{M}$ : Capsaicin exhibits neuroprotective effects in a model of transient global cerebral ischemia in Mongolian gerbils. Br J Pharmacol 2005, 144:727-735.

15. Zhang M, Martin BR, Adler MW, Razdan RK, Ganea D, Tuma RF Modulation of the balance between cannabinoid $\mathrm{CB}(1)$ and $\mathrm{CB}(2)$ receptor activation during cerebral ischemic/reperfusion injury. Neuroscience 2008, 152:753-760.

16. Ashton JC, Rahman RMA, Nair SM, Sutherland BA, Glass M, Appleton I: Cerebral hypoxia-ischemia and middle cerebral artery occlusion induce expression of the cannabinoid CB2 receptor in the brain. Neurosci Lett 2007, 412:114-117.

17. Saleh A, Schroeter M, Ringelstein A, Hartung HP, Siebler M, Modder U, Jander S: Iron oxide particle-enhanced MRI suggests variability of brain inflammation at early stages after ischemic stroke. Stroke 2007, 38:2733-2737

18. Zhang M, Martin BR, Adler MW, Razdan RK, Jallo Jl, Tuma RF: Cannabinoid $\mathrm{CB}(2)$ receptor activation decreases cerebral infarction in a mouse focal ischemia/reperfusion model. J Cereb Blood Flow Metab 2007, 27:1387-1396

19. Schäbitz WR, Giuffrida A, Berger C, Aschoff A, Schwaninger M, Schwab S, Piomelli D: Release of fatty acid amides in a patient with hemispheric stroke. Stroke 2002, 33:2112-2114.

20. Brott T, Adams HP, Olinger CP, Marler JR, Barsan WG, Biller J, Spilker J, Holleran R, Eberle R, Hertzberg V, Rorick M, Moomaw CJ, Walker M: Measurements of acute cerebral infarction: a clinical examination scale. Stroke 1989, 20:864-870

21. Rödén-Jüllig Å, Gustafsson C, Fugl-Meyer A: Validation of four scales for the acute stage of stroke. J Internal Med 1994, 236:125-136.

22. De Marchi N, De Petrocellis L, Orlando P, Daniele F, Fezza F, Di Marzo V Endocannabinoid signalling in the blood of patients with schizophrenia. Lipids Health Dis 2003, 2:5.

23. Monteleone P, Matias I, Martiadis V, De Petrocellis L, Maj M, Di Marzo V: Blood levels of the endocannabinoid anandamide are increased in anorexia nervosa and in binge-eating disorder, but not in bulimia nervosa. Neuropsychopharmacology 2005, 30:1216-1221.

24. Bluher M, Engeli S, Kloting N, Berndt J, Fasshauer M, Batkai S, Pacher P, Schon MR, Jordan J, Stumvoll M: Dysregulation of the peripheral and adipose tissue endocannabinoid system in human abdominal obesity. Diabetes 2006, 55:3053-3060.

25. Zolese G, Bacchetti T, Ambrosini A, Wozniak M, Bertoli E, Ferretti G: Increased plasma concentrations of Palmitoylethanolamide, an endogenous fatty acid amide, affect oxidative damage of human lowdensity lipoproteins: An in vitro study. Atherosclerosis 2005, 182:47-55.

26. Di Marzo V: Targeting the endocannabinoid system: to enhance or reduce? Nat Rev Drug Discov 2008, 7:438-455.
27. Centonze D, Bari M, Rossi S, Prosperetti C, Furlan R, Fezza F, De Chiara V, Battistini L, Bernardi G, Bernardini S, Martino G, Maccarrone M: The endocannabinoid system is dysregulated in multiple sclerosis and in experimental autoimmune encephalomyelitis. Brain 2007, 130:2543-2553.

28. Battista N, Bari M, Tarditi A, Mariotti C, Bachoud-Lévi AC, Zuccato C, Finazzi-Agrò A, Genitrini S, Peschanski M, Di Donato S, Cattaneo E, Maccarrone M: Severe deficiency of the fatty acid amide hydrolase (FAAH) activity segregates with the Huntington's disease mutation in peripheral lymphocytes. Neurobiol Dis 2007, 27:108-116.

29. Cupini LM, Bari M, Battista N, Argirò G, Finazzi-Agrò A, Calabresi P, Maccarrone M: Biochemical changes in endocannabinoid system are espresse in platelets of female but not male migraineurs. Cephalalgia 2006, 26:277-281

30. Rossi C, Pini LA, Cupini ML, Calabresi P, Sarchielli P: Endocannabinoids in platelets of chronic migraine patients and medication-overuse headache patients: relation with serotonin levels. Eur J Clin Pharmacol 2008, 64:1-8.

31. Hill MN, Miller GE, Ho WS, Gorzalka BB, Hillard CJ: Serum endocannabinoid contentis altered in females with depressive disorders: a preliminary report. Pharmacopsychiatry 2008, 41:48-53.

32. Centonze D, Battistini L, Maccarrone M: The endocannabinoid system in peripheral lymphocytes as a mirror of neuroinflammatory diseases. Curr Pharm Des 2008, 14:2370-2442.

33. Pellegrini-Giampietro DE, Mannaioni G, Bagetta G: Post-ischemic brain damage: the endocannabinoid system in the mechanisms of neuronal death. FEBS Journal 2009, 276:2-12.

34. Maccarrone M, Rossi S, Bari M, De Chiara V, Fezza F, Musella A, Gasperi V Prosperetti C, Bernardi G, Finazzi-Agrò A, Cravatt BF, Centonze D: Anandamide inhibits metabolism and physiological actions of 2arachidonoylglycerol in the striatum. Nat Neurosci 2008, 11:152-159.

35. Re G, Barbero R, Miolo A, Di Marzo V: Palmitoylethanolamide, endocannabinoids and related cannabimimetic compounds in protection against tissue inflammation and pain: potential use in companion animals. Vet J 2007, 173:21-30

36. Mechoulam R, Shohami E: Endocannabinoids and traumatic brain injury. Mol Neurobiol 2007, 36:68-74.

doi: 10.1186/1476-511X-9-47

Cite this article as: Naccarato et al., Possible Anandamide and Palmitoylethanolamide involvement in human stroke Lipids in Health and Disease 2010, 9:47

\section{Submit your next manuscript to BioMed Central and take full advantage of:}

- Convenient online submission

- Thorough peer review

- No space constraints or color figure charges

- Immediate publication on acceptance

- Inclusion in PubMed, CAS, Scopus and Google Scholar

- Research which is freely available for redistribution 